\title{
Kaplan's Lesion of the Little Finger Treated with Open Reduction by a Volar Approach: A Case Report
}

\author{
Jung Hwan Um (D), Soon Heum Kim (D), Dong In Jo (D) \\ Department of Plastic and Reconstructive Surgery, Konkuk University Chungju Hospital, Konkuk University School of Medicine, Chungju, Korea
}

\begin{abstract}
Kaplan's lesions are defined as open wounds with the metacarpal head exposed in the palms, accompanied by complex dorsal dislocation of the metacarpophalangeal joint (MCPJ). Kaplan's lesions are clinically rare because the volar side of the MCPJ is anatomically supported and reinforced by a stronger adjacent structure. Moreover, lesions in the little finger are very rarely reported because most Kaplan's lesions occur in the index finger. The reduction of lesions and restoration of joint stability is difficult when Kaplan's lesions occur. Various methods have been currently introduced in the treatment of Kaplan's lesions; however, no standardized treatment has been established because of the rarity of this disease. This paper reports a case of Kaplan's lesion of the left little finger without fracture after a fall; the case was successfully treated with open reduction using a volar approach.
\end{abstract}

Keywords: Kaplan's lesion; Joint dislocation; Metacarpophalangeal joint; Little finger; Trauma

\section{Introduction}

Kaplan's lesions are defined as open wounds of metacarpal head buttonholes exposed into the palms, accompanied by complex dorsal dislocation of the metacarpophalangeal joint (MCPJ) [1,2]. These injuries are clinically rare [3]. Studies have reported that they are caused by forceful hyperextension of the MCPJ. The index finger is the most common digit involved, followed by the thumb. Complex dorsal dislocation of the MCPJ in the little finger is very rare $[4,5]$.

When a Kaplan's lesion occurs, it is easy to assume that it can be treated with closed reduction alone followed by skin closure; however, in reality closed reduction is difficult and nearly impossible [6]. Although the volar side of the MCPJ is protected by thick and sturdy adjacent structures, the possibility of these structures rupturing in case of complex dorsal dislocation of the MCPJ must be considered. Moreover, although rare, the sesamoid bones between the MCPJs may be buried in the volar plate, indicating that the anatomical location should be considered when performing reduction. For these reasons, the surgeon should consider treatment methods that allow restoration of joint stability as well as reduction in Kaplan's lesions. Currently, various surgical methods, such as the volar approach, dorsal approach, and lateral approach, have been introduced in the treatment of Kaplan's lesions, but no standardized treatment has been established due to their rare occurrence [1,7]. In case of complex dorsal dislocation of the MCPJ in the little finger, the abductor digiti minimi $(\mathrm{ADM})$ and flexor digiti minimi (FDM) muscles are found at the lateral border of the

\section{Case Report}

Received: March 11, 2021

Revised: May 21, 2021

Accepted: May 22, 2021

\section{Corresponding author:}

Dong In Jo, M.D., Ph.D.

Department of Plastic and Reconstructive Surgery, Konkuk University Chungju Hospital, Konkuk University School of Medicine, 82 Gugwon-daero, Chungju 27376, Korea Tel: +82-43-840-8860

Fax: +82-43-840-9862

E-mail: cozarmd@kku.ac.kr

This is an Open Access article distributed under the terms of the Creative Commons Attribution Non-Commercial License (https://creativecommons.org/licenses/by-nc/4.0/) which permits unrestricted non-commercial use, distribution, and reproduction in any medium, provided the original work is properly cited.

C 2021 Korean Wound Management Society 
MCPJ instead of lumbrical muscles, indicating an anatomical difference from the relatively frequently reported cases affecting the index finger and thumb.

This study reports a case of Kaplan's lesion of the little finger treated with open reduction using the volar approach. The study was approved by the Institutional Review Board of the Konkuk University Chungju Hospital (IRB No. KUCH 202101-001). The patient provided written informed consent for the publication and use of his images.

\section{Case}

A 61-year-old male with a history of stroke presented to the emergency room after a fall from a height of $2 \mathrm{~m}$ at work. The patient was diagnosed with an L2 spine compression fracture on lumbar computed tomography, and a 4-cm horizontal lac-
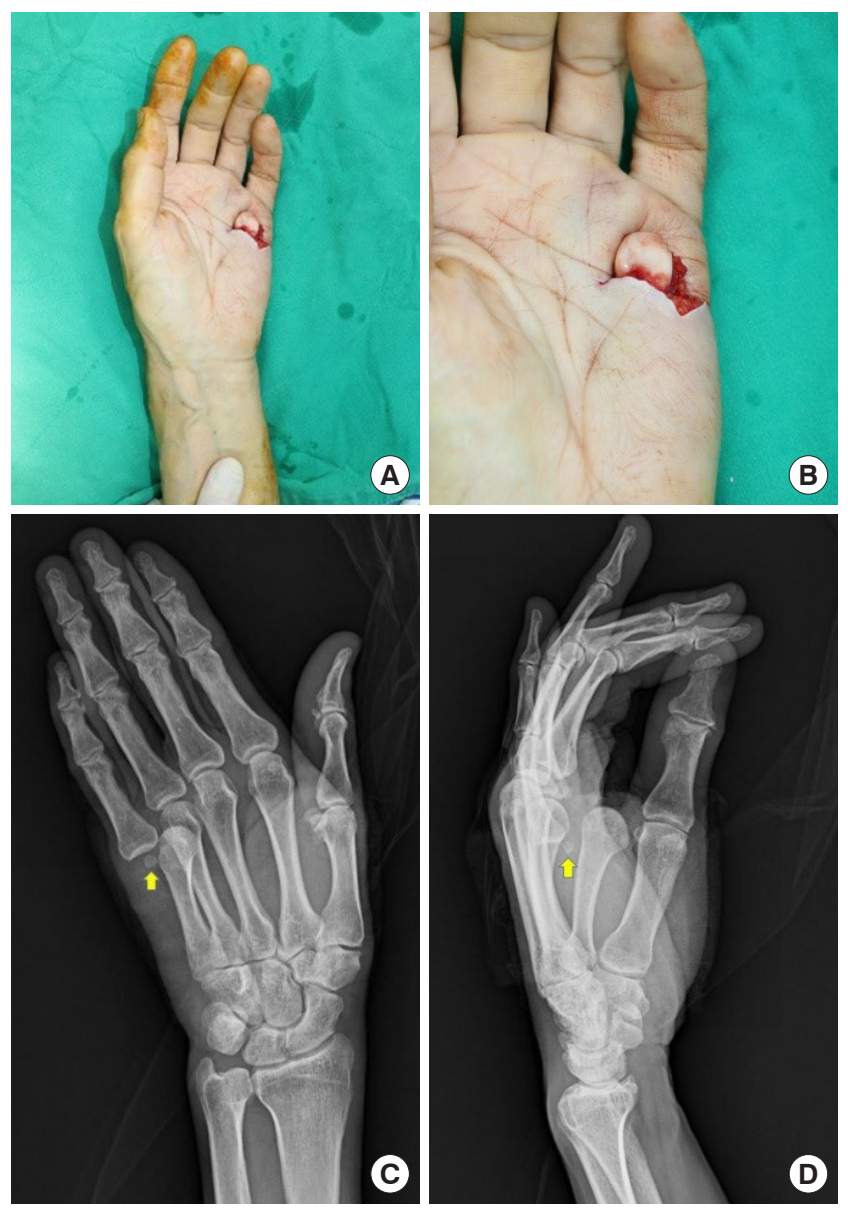

Fig. 1. A patient presented with dislocation of the left little finger. Clinical photographs before surgery; (A) palmar view and (B) focus view. Preoperative X-ray; (C) anteroposterior view and (D) lateral view. Yellow arrow is the sesamoid bone. eration was observed on the volar side of the MCPJ of the little finger of his left hand (Fig. 1A). The metacarpal bone head was found dislocated in the open wound (Fig. 1B). The patient could move the distal and proximal interphalangeal joints of his left little finger, and the sensation of the finger was also preserved. A hand X-ray revealed a complex dorsal dislocation of the MCPJ of the left little finger but no fracture (Fig. 1C and D). Though closed reduction was performed, it was not successful because of the imposing tissue. Thus, open reduction was planned under brachial plexus block anesthesia.

An extended horizontal incision and an additional vertical incision, both $2 \mathrm{~cm}$ long, were made on the head of the metacarpal bone within the horizontal open wound (Fig. 2A). When the distal border of the open wound was elevated, the natatory ligament was observed, and superficial transverse ligament was found with the elevation of the proximal border. Muscle bellies of the ADM and FDM were observed at the ulnar border of the head of the fifth metacarpal bone, and the flexor tendon of the little finger was observed at the radial border. No fracture or tendon and vessel injuries were found upon further exploration. The ruptured volar plate was observed in the dislocated space, and a 3-mm sesamoid bone was attached to the proximal end of the ruptured volar plate. After removing the entrapped ruptured volar plates, the proximal phalanx was reduced and the ruptured volar plates were reconstructed. At this time, the sesamoid bone was buried under the reconstructed volar plate. The volar plate and subcutaneous tissue were reconstructed using Vicryl. The operation was concluded after closing the skin with nylon sutures (Fig. 2B). An ulnar gutter splint was used to minimize joint movement for 14 days,
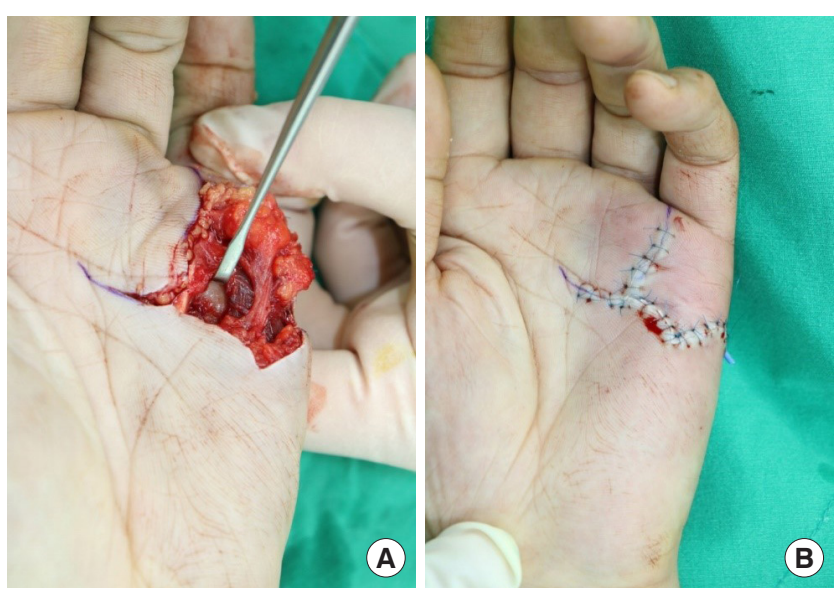

Fig. 2. Open reduction by a volar approach was performed. (A, B) Intraoperative findings; palmar view. 

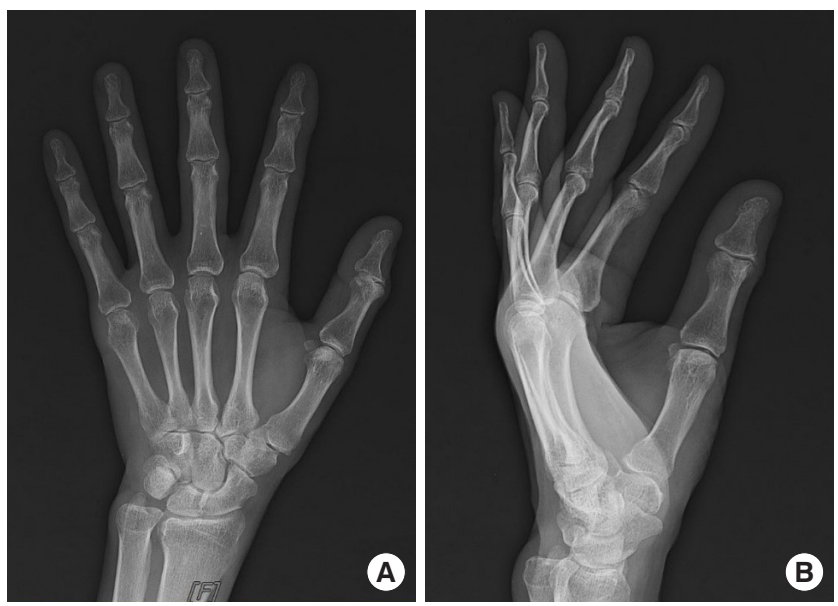

Fig. 3. No redislocation was noted after reduction on the postoperative X-ray. (A) Anteroposterior view and (B) lateral view.

after which hand rehabilitation was initiated. No redislocation was noted after reduction on postoperative X-ray (Fig. 3).

Upon assessment of the left fifth MCPJ on postoperative day 5 , smooth flexion and extension movement were observed. To avoid possible damage to the operative site, the range of motion (ROM) of the finger was not evaluated. At the second postoperative week, no dysesthesia of the left little finger was observed. The ROM of the repaired MCPJ was evaluated on follow-up 6 months postoperatively. The flexion and extension angles of the MCPJ were $82^{\circ}$ and $-4^{\circ}$, respectively. This indicated that more than $70 \%$ of the normal value of ROM as per the American Medical Association criteria was recovered (Fig. $4 \mathrm{~A}$ and B) [8]. Moreover, no abnormal scars, such as skin atrophy or hypertrophic lesions were observed (Fig. 4C). Followup was discontinued since then.

\section{Discussion}

Kaplan's lesions are caused by the rupture of the volar plate, comprised of thick and sturdy tissue, in a hyperextended MCPJ, making such lesions rare. Approximately $50 \%$ of all Kaplan's lesions are accompanied with a fracture of the metacarpophalangeal head [7,9]. Kaplan's lesions often affect the index finger, thumb, and little finger, in this order. Given the support of the neighboring digits and deep transverse metacarpal ligaments, injuries of the middle and ring fingers are rare [10]. In general, MCPJ dislocation is associated with the joint and adjacent supporting structures surrounding the joint. MCPJ is a condyloid joint, and the head of the metacarpal bone, which widens from the dorsal side to the volar side, increases the

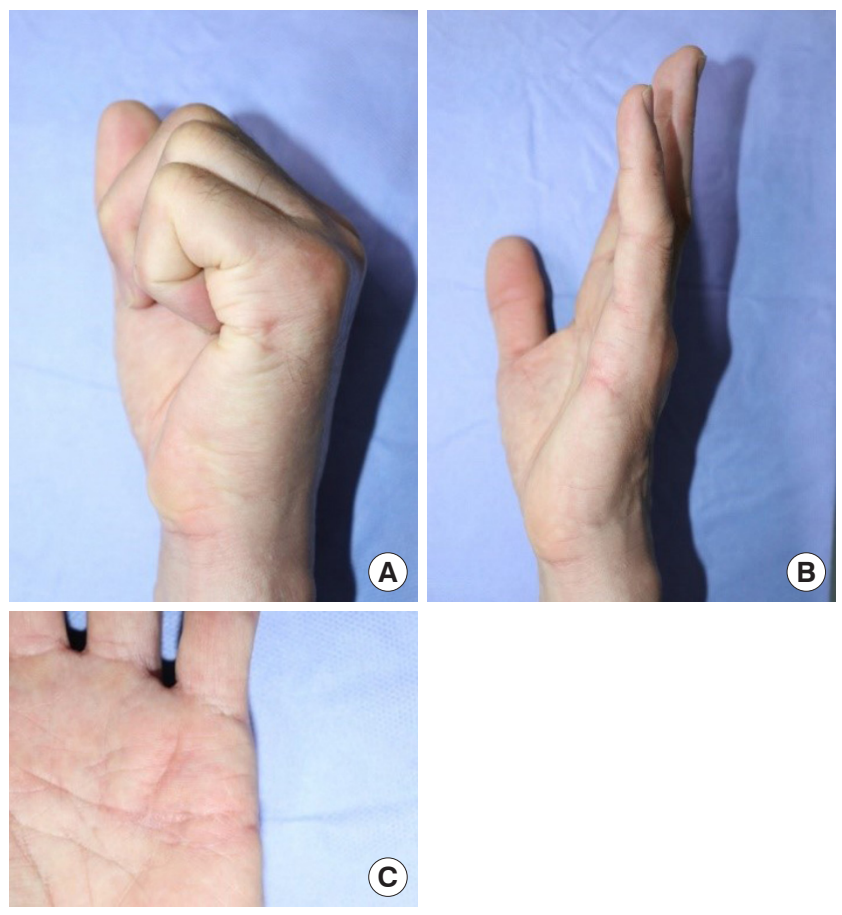

Fig. 4. The range of motion was evaluated at postoperative month 6. Clinical photographs 6 months later; (A) flexion, (B) extension, and (C) a scar.

contact surface with the base of the proximal phalanx with the MCPJ flexion. The volar side of the joint capsule is also supported by the volar plate connecting with fibrocartilaginous tissues, such as the sheath of the flexor tendon, natatory ligament, and superficial transverse ligament [6].

The volar plate plays a role in the stability and rigid support of the MCPJ on the palm surface. Moreover, the flexor tendon and A1 pulley are located on the volar side of the volar plate, providing additional stability to the MCPJ [6,9]. Despite these anatomical features, the structure of the volar plate was damaged in this case due to MCPJ hyperextension as the patient fell. MCPJ dislocation may have occurred due to increased joint instability.

The noose theory suggests that closed reduction is difficult to perform in cases with Kaplan's lesions. It is hypothesized that the head of the metacarpal bone, which is dislocated and observed within the open wound, is noosed by adjacent structures (e.g., the natatory ligament, superficial transverse metacarpal ligament, flexor tendons, pretendinous band, and lumbricals) $[1,11]$. In case of Kaplan's lesions that affect the little finger, as in this case, this noose theory is modified to accommodate the anatomical differences. In the little finger, there are no lumbrical muscles at the ulnar border of the MCPJ but 


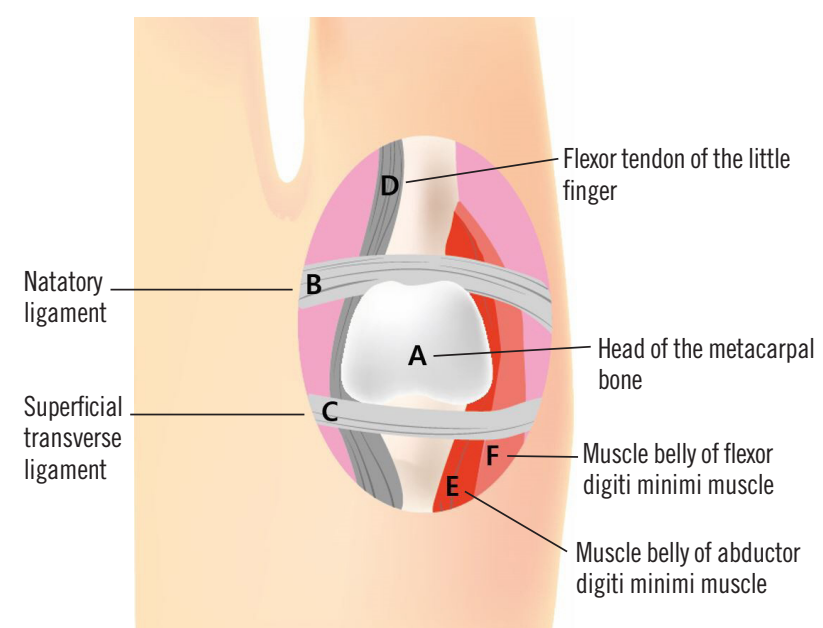

Fig. 5. Illustration of Kaplan's lesion of the little finger; palmar view.

there are ADM and FDM; these muscles are attached to the ulnar and volar sides of the proximal phalanx, respectively (Fig. 5). Therefore, the surgeon should be aware of these anatomical differences and be more attentive to possible damage to the ADM and FDM muscle belly during reconstruction.

When planning treatment for Kaplan's lesions, the surgeon should aim at the restoration of the patient's MCPJ motion and perform the reduction and repair of the volar plate and surrounding soft tissues. In case of excessive closed reduction, the interposition of the volar plate and articular capsule occurs on both the volar and dorsal sides. According to previous studies, few cases of successful closed reduction exist because the volar plate, capsule joint, and collateral ligaments imposing on the joint are entrapped in the joint and thus interfere with the reduction [12].

The most important point of the surgical treatment of Kaplan's lesions is to remove the imposing structures in the dislocated space and release the metacarpal head from the noose formed by the adjacent structures. The preferred method for this surgical treatment has changed over time. The dorsal approach was first introduced in 1876 , enabling easy access to associated osteochondral fractures and entrapped volar plates as well as decreasing the risk of neurovascular injury. However, the anatomical restoration of the entrapped volar plate and damaged adjacent structures is difficult using this method, and MCPJ instability remains [13]. In contrast, the volar approach enables access not only to the volar plate but also to adjacent structures noosing the metacarpal head due to the excellent visualization provided by the volar side, unlike the dorsal approach. This makes it possible to reconstruct the anatomically complete volar plate and adjacent structures, thereby restoring the stability of the MCPJ. As a modified volar-approach method, the A1 pulley is released to decrease the tension on the flexor tendon so the volar plate returns to its original anatomical position [9]. However, the volar approach is associated with a high risk of neurovascular injury. Because the displaced metacarpal head can move the neurovascular bundle upward under the skin, this possibility should be considered when using the volar approach. Recently, a lateral approach that can expose both volar and dorsal structures has been introduced. This approach allows the visualization and treatment of both volar and dorsal structures [7]. Moreover, it is easy to reduce osteochondral fractures in the dorsal side of the metacarpal head as well as to re-insert the volar plate. This method also minimizes the limitation to joint motion caused by surgical scars because the MCPJ is a condyloid joint. However, this approach is associated with digital nerve and vessel damage.

In this case, the authors confidently used the volar approach for the following reasons: first, the traumatic wound was not accompanied by a fracture; second, the open wound was already large enough to approach, and third, the anatomical location of the sesamoid bone was clearly visible on the preoperative $\mathrm{X}$-ray. This made it easy to access the lesion and thereby provided good results, including recovery of motor function. If the sesamoid bone is not clearly visible in the preoperative $\mathrm{X}$-ray view, or if the positional relationship between the bones is inaccurate due to fractures, preoperative computed tomography is recommended [5].

Standard treatment for Kaplan's lesion has not yet been established as such lesions are rare; however, various approaches can be applied depending on the traumatic condition of the lesion and the surgeon's preference. Based on anatomical differences, the surgeon should consider the restoration of the ruptured volar plate and adjacent structures as well as the reduction of the complex dorsal dislocation of the MCPJ of the little finger.

\section{Conflict of interest}

No potential conflict of interest relevant to this article was reported.

\section{ORCID iDs}

Jung Hwan Um

Soon Heum Kim https://orcid.org/0000-0002-9129-5582

https://orcid.org/0000-0001-9773-4753 
Um JH et al.

Kaplan's lesion of the little finger

Dong In Jo ～https://orcid.org/0000-0002-3075-4482

\section{References}

1. Kaplan EB. Dorsal dislocation of the metacarpophalangeal joint of the index finger. J Bone Joint Surg Am 1957;39A:1081-6.

2. Kaplan EB, Spinner M. Kaplan's functional and surgical anatomy of the hand. 3rd ed. Philadelphia: Lippincott Williams \& Wilkins; 1984.

3. Green DP, Terry GC. Complex dislocation of the metacarpophalangeal joint: correlative pathological anatomy. J Bone Joint Surg Am 1973;55:1480-6.

4. Patterson RW, Maschke SD, Evans PJ, et al. Dorsal approach for open reduction of complex metacarpophalangeal joint dislocations. Orthopedics 2008;31:1099.

5. Elghoul N, Bouya A, Jalal Y, et al. Complex metacarpophalangeal joint dislocation of the litter finger: a sesamoid bone seen within joint. What does it mean? Trauma Case Rep 2019;23:100225.

6. Eaton RG, Dray GJ. Dislocations and ligament injuries in the digits. In: Green DP, editor. Green's operative hand surgery. New York: Churchill Livingstone; 1982. p. 647-68.

7. Pereira JM, Quesado M, Silva M, et al. The lateral approach in the surgical treatment of a complex dorsal metacarpophalangeal joint dislocation of the index finger. Case Rep Orthop 2019;2019:1063829.

8. Gerhardt JJ, Cocchiarella L, Lea RD, et al. The practical guide to range of motion assessment. Chicago: American Medical Association; 2002.

9. Dinh P, Franklin A, Hutchinson B, et al. Metacarpophalangeal joint dislocation. J Am Acad Orthop Surg 2009;17: 318-24.

10. May JW Jr, Rohrich RJ, Sheppard J. Closed complex dorsal dislocation of the middle finger metacarpophalangeal joint: anatomic considerations and treatment. Plast Reconstr Surg 1988;82:690-3.

11. Afifi AM, Medoro A, Salas C, et al. A cadaver model that investigates irreducible metacarpophalangeal joint dislocation. J Hand Surg Am 2009;34:1506-11.

12. Patel MR, Bassini L. Irreducible palmar metacarpophalangeal joint dislocation due to junctura tendinum interposition: a case report and review of the literature. J Hand Surg Am 2000;25:166-72.

13. Durakbasa O, Guneri B. The volar surgical approach in complex dorsal metacarpophalangeal dislocations. Injury 2009;40:657-9. 